J. Asiat. Soc. Bangladesh, Sci. 44(1): 7-14, June 2018

\title{
NEONATAL, INFANT AND UNDER-FIVE MORTALITY: AN APPLICATION OF COX PROPORTIONAL HAZARD MODEL TO BDHS DATA
}

\author{
SHAHNAZ NILIMA ${ }^{1 *}$, REBEKA SULTANA ${ }^{2}$ AND SHAHJADI IREEN ${ }^{2}$ \\ ${ }^{I}$ Department of Statistics, University of Dhaka \\ ${ }^{2}$ Department of Statistics, Jagannath University, Dhaka, Bangladesh
}

\begin{abstract}
This study utilizes data derived from the Bangladesh demographic and health survey (BDHS), 2014 to identify the determinants of neonatal, infant and under-five mortality in Bangladesh. Log-rank test has been used for bivariate analysis. Regression analysis has been performed by applying Cox proportional hazard model to the data. It has been found from the analysis that maternal education, region, exposure to NGO activities are significant determinants of under-five and infant mortality whereas region, gender of child, child's size at birth play significant role in reducing neonatal mortality in Bangladesh. The findings of the study suggest that policymakers should give priority on maternal education, region, and child's size at birth as well as exposure to NGO activities to reduce neonatal, infant and under-five mortality in Bangladesh.
\end{abstract}

Key words: Under-five mortality, Infant mortality, Neonatal mortality, Log-rank test, Cox Proportional Hazard model

\section{Introduction}

Child mortality is an important public health issue throughout the world since it is commonly used as one of the important measures of well-being and development of a country. Mortality of child is defined distinctly with respect to the age of a child as neonatal, infant and under-five mortality. By definition, neonatal mortality includes the deaths within first month of life, infant mortality includes the deaths within first year of life and under-five mortality includes the deaths between birth and fifth birthday (BDHS 2014). According to World Health Organization (WHO), around 5.6 million children died worldwide before reaching their fifth birthday in 2016. Globally remarkable progress has been made in child survival by reducing under-five mortality rate by 53 per cent from 91 deaths per 1000 live births in 1990 to 43 deaths per 1000 live births in 2015 (UNICEF 2015). Like other developing countries, Bangladesh has achieved Millennium Development Goal (MDG) 4 target of two-thirds reduction of under-five mortality rate from 133 deaths per 1000 live births in 1989 - 1993 to 46 deaths per 1000 live births in 2010 - 2014 (BDHS 2014). The infant mortality rate is 38 per 1000 live births in Bangladesh. Over the last two decades, infant mortality rate has been dropped by 56 per

\footnotetext{
*Author of correspondence. Email: s.nilima_du@yahoo.com
} 
cent from 87 to 38 deaths per 1000 live births (BDHS 2014). Although infant mortality rate has been declined, it has been observed that infant mortality comprised of $65 \%$ of under-five deaths in 1989 - 1993 which raised to 83\% in 2010 - 2014. Reducing underfive mortality to 37 or fewer deaths per 1000 live births by 2021 in Bangladesh is one of the targets of the health, nutrition and population sector program (MOHFW 2016). As the largest portion of under-five deaths took place during infancy, it becomes more important to identify the factors affecting infant mortality for reducing under-five mortality to achieve this target. The BDHS (2014) data reveal that neonatal mortality rate is 28 per 1000 live births indicating 46\% fall in neonatal mortality compared to the period 1989 1993. Although under-five and infant mortality rates have declined, $61 \%$ of under-five deaths and $74 \%$ of infant deaths occurred in the neonatal period of life. It is expected to focus on neonatal mortality in order to decrease under-five and infant mortality rates. It is also notable that the pace of reduction in neonatal mortality (46\%) from 1989 to 2014 is slowest compared with under-five (65\%) and infant mortality (56\%) (BDHS 2014). The pace of reduction in neonatal deaths should be accelerated to achieve the target of the health, nutrition and population sector program of 21 neonatal deaths per 1000 live births by 2021 (MOHFW 2016). The third sustainable development goal (SDG) is reducing neonatal mortality rate to at least as low as 12 deaths per 1,000 live births and under-five mortality to at least as low as 25 deaths per 1,000 live births. To achieve SDG 3 target, it is important to identify the factors influencing the under-five and neonatal mortality in Bangladesh. To ensure better development and well-being of the country, it is needed to improve child health by reducing neonatal, infant and under-five mortalities.

Globally social scientists and policymakers are greatly interested on the factors affecting child mortality as it is considered as an indicator of standard of living and socioeconomic development of a nation. Worldwide various studies have been conducted to find the determinants of neonatal, infant and under-five mortality. Nisar and Dibley (2014) performed Cox regression analysis to determine the risk factors of neonatal mortality in Pakistan. In Nepal, Khadka et al. (2015) investigated the socio-economic determinants of infant mortality using logistic regression. A study was undertaken by Nasejje et al. (2015) to examine the determinants of under-five mortality in Uganda.

In Bangladesh, several studies have been conducted to identify the determinants of neonatal, infant and under-five mortality to promote socio-economic development and quality of life. Chowdhury (2013) performed Cox Proportional Hazard analysis to find the determinants of under-five mortality in Bangladesh. Place of residence, region and mother's age have been found significant factors affecting under-five mortality in his study. Mondal et al. (2009) investigated the factors having significant impact on infant and child mortality at Rajshahi district. The findings of the study revealed that the most 
significant determinant of neonatal, post-neonatal and child mortality was mother's age at birth. Mother's education and treatment places were significant predictors of both neonatal and child mortality whereas residence, watching television, sex of child and birth order were found significant only for child mortality. Kamal et al. (2012) found maternal age, maternal education and birth order as potential risk factors of neonatal mortality in Bangladesh. Hossain and Islam (2008) studied the socio-economic variables affecting infants and children mortality. In this study, it was observed that mother's education, medical checkup during pregnancy and watches TV were significant factors influencing infant, child and under-five mortality. Another study on infant mortality by Fatima et al. (2016) used log-logistic parametric survival regression model and revealed that age of mother at birth, maternal education, gender of child, size of child at birth, place of delivery and membership of NGO significantly influence the infant mortality. Rahman (2008) conducted a study using Cox proportional hazard model to BDHS data and found that place of delivery, maternal education, source of drinking water, household electricity facility and household assets index had significant role in declining the child mortality rate.

In this paper an attempt has been made to determine the potential determinants affecting neonatal, infant and under-five mortality in Bangladesh.

\section{Materials and Methods}

The study uses secondary data extracted from nationally representative Bangladesh Demographic and Health Survey (BDHS) conducted in 2014 under the authority of the National Institute of Population Research and Training (NIPORT) of the Ministry of Health and Family Welfare. This survey interviewed 17,863 ever married women aged 15 - 49 and asked to provide information of their live births including the sex, month, year of each birth, survival status and age at the time of the survey or age at death and also asked about background characteristics (e.g., age, education, region, media exposure etc.).

Three outcome variables were considered in this study: the time to death within five years of a child; the time to death before reaching it's first birthday and the time to death within one month of life. A child is considered to be censored if it did not die within neonatal and infancy period and before reaching it's fifth birthday.

Based on previous studies (Chowdhury 2013, Mondal et al. 2009, Hossain and Islam 2008, Rahman 2008, Siddiqi et al. 2011, Kamal et al. 2012, Fatima et al. 2016) a set of explanatory variables are considered in this study which are mother's age at birth, maternal education, place of residence, region, wealth index, birth order number, gender of the child, place of delivery, child's size at birth, exposure to NGO (Non Government Organizations) activities and Access to media. 
In order to identify the determinants affecting childhood mortality, information from 4723 children was used for our study purpose. Among these children, 170 died before fifth year of their birth, 163 died before first year of their birth and 125 died before first month of their birth which yields under-five mortality, infant mortality and neonatal mortality rates are 36, 35 and 26 deaths per 1000 live births, respectively. These rates underestimate the under-five mortality rate (46 per 1000 live births), infant mortality rate (38 per 1000 live births), and neonatal mortality rate (28 per 1000 live births) presented in BDHS (2014) report. This may occur because we deleted 3158 missing cases for child's size at birth and 3,162 missing cases for place of delivery and also excluded those children, who died before reaching their fifth birthday but they were born before preceding five years of the survey.

Log-rank test and Cox Proportional Hazard Model are used for bivariate and multivariate regression analysis, respectively.

\section{Results and Discussion}

Bivariate analysis: Table 1 presents the $\mathrm{p}$ values obtained from log-rank test for underfive, infant and neonatal mortality for selected explanatory variables. It has been found that maternal education, region, child's size at birth, exposure to NGO activities and access to media are significantly associated with under-five, infant and neonatal mortality. Besides those variables, wealth index shows significant relation with underfive mortality and gender of child is associated with neonatal mortality. The variables that are found significant are considered for Cox Proportional regression analysis.

Table 1. $\mathrm{P}$ values obtained from Log-rank test for under-five, infant and neonatal mortality for the selected explanatory variables.

\begin{tabular}{lccc}
\hline \multirow{2}{*}{ Variables } & \multicolumn{3}{c}{ Log rank test p values } \\
\cline { 2 - 4 } & Under-five mortality & Infant mortality & Neonatal mortality \\
\hline Mother's age at birth & 0.693 & 0.680 & 0.650 \\
Maternal education & $\mathbf{0 . 0 0 6}$ & $\mathbf{0 . 0 1 0}$ & $\mathbf{0 . 0 6 0}$ \\
Place of residence & 0.580 & 0.850 & 0.710 \\
Region & $\mathbf{0 . 0 0 5}$ & $\mathbf{0 . 0 0 4}$ & $\mathbf{0 . 0 0 6}$ \\
Wealth index & $\mathbf{0 . 0 5 0}$ & 0.140 & 0.380 \\
Birth order number & 0.410 & 0.260 & 0.400 \\
Gender of child & 0.300 & 0.330 & $\mathbf{0 . 0 6 0}$ \\
Place of delivery & 0.510 & 0.670 & 0.710 \\
Child's size at birth & $\mathbf{0 . 0 5 0}$ & $\mathbf{0 . 0 4 0}$ & $\mathbf{0 . 0 3 0}$ \\
Exposure to NGO activities & $\mathbf{0 . 0 6 0}$ & $\mathbf{0 . 0 4 0}$ & $\mathbf{0 . 0 6 0}$ \\
Access to media & $\mathbf{0 . 0 1 0}$ & $\mathbf{0 . 0 4 0}$ & $\mathbf{0 . 0 6 0}$ \\
\hline
\end{tabular}


Survival regression analysis: The hazard ratios and $\mathrm{p}$ values obtained applying Cox proportional hazard model to under-five, infant and neonatal mortality data are reported in Table 2.

Under-five mortality: The study shows that the hazard rate of under-five mortality among children with higher educated mothers is $57 \%$ lower than that of children with illiterate mothers. The results reveal that maternal education is negatively associated with under-five mortality which found similar to the findings of the previous studies (Siddiqi et al. 2011, Islam et al. 2013). This may happen because education enhances mothers' awareness about child health, child nutrition and modern child health facilities. Table 2 depicts that the children of Sylhet experience the highest rate of mortality compared to the children of Dhaka. It is also observed that children who belong to Barisal experience the lowest rate of mortality compared to the children of Dhaka which conflicts with the results of the study of Chowdhury (2013). It is clear from Table 2, mother's exposure to NGO activities shows significant association with under-five mortality. A child whose mother is exposed to NGO activities experiences $33 \%$ more rate of underfive mortality compared to the child whose mother is not exposed to NGO. It may be due to the fact that the mothers who are engaged with an NGO do not get enough time to take proper care of their children. The variables wealth index, child's size at birth and access to media have been found insignificant factors determining the under-five mortality.

Infant mortality: The analysis reveals that children of higher educated mothers are more likely to survive during infancy compared to the infants of mothers who didn't attend school. Therefore, maternal education shows significant inverse relation with infant mortality which were similar to the study of Fatima et al. (2016). The study demonstrates that the rate of dying within first year of life is highest among children of Sylhet region. It has been found interesting that infants of Barisal have lower rate of mortality compared to that of Dhaka which contradicts with the results of the former study conducted in Bangladesh (Fatima et al. 2016). It is clear from Table 2 that children of mothers affiliated to NGO activities experience higher rate of dying during infancy than that of mothers who are not affiliated with NGO. Child's size at birth and access to media have no significant effects on infant mortality like under-five mortality. 
Table 2. Hazard ratios and $p$ values obtained from Cox Proportional Hazard analysis for under-five, infant and neonatal mortality for the selected explanatory variables.

\begin{tabular}{|c|c|c|c|c|c|c|}
\hline \multirow[t]{2}{*}{ Variables } & \multicolumn{2}{|c|}{ Under-five mortality } & \multicolumn{2}{|c|}{$\begin{array}{c}\text { Infant } \\
\text { mortality }\end{array}$} & \multicolumn{2}{|c|}{$\begin{array}{l}\text { Neonatal } \\
\text { mortality }\end{array}$} \\
\hline & $\mathrm{HR}$ & p-value & $\mathrm{HR}$ & $\mathrm{p}$-value & HR & p-value \\
\hline \multicolumn{7}{|l|}{ Maternal education } \\
\hline No education(RC) & - & - & - & - & - & - \\
\hline Primary & 0.977 & 0.92 & 1.051 & 0.83 & 1.138 & 0.63 \\
\hline Secondary & 0.867 & 0.54 & 0.895 & 0.64 & 1.007 & 0.97 \\
\hline Higher & 0.425 & 0.04 & 0.462 & 0.07 & 0.490 & 0.14 \\
\hline \multicolumn{7}{|l|}{ Region } \\
\hline Dhaka(RC) & - & - & - & - & - & - \\
\hline Barisal & 0.404 & 0.02 & 0.367 & 0.02 & 0.404 & 0.07 \\
\hline Chittagong & 0.950 & 0.85 & 0.945 & 0.83 & 0.989 & 0.97 \\
\hline Khulna & 1.276 & 0.38 & 1.190 & 0.55 & 1.514 & 0.22 \\
\hline Rajshahi & 1.058 & 0.85 & 1.020 & 0.92 & 1.451 & 0.27 \\
\hline Rangpur & 0.837 & 0.57 & 0.870 & 0.65 & 1.204 & 0.60 \\
\hline Sylhet & 1.522 & 0.09 & 1.569 & 0.07 & 1.884 & 0.03 \\
\hline \multicolumn{7}{|l|}{ Wealth index } \\
\hline Poor & 1.243 & 0.36 & & & & \\
\hline Middle (RC) & - & - & & & & \\
\hline Rich & 1.219 & 0.41 & & & & \\
\hline \multicolumn{7}{|l|}{ Gender of child } \\
\hline Male (RC) & & & & & - & - \\
\hline Female & & & & & 0.689 & 0.04 \\
\hline \multicolumn{7}{|l|}{ Child's size at birth } \\
\hline Large/Average (RC) & - & - & - & - & - & - \\
\hline Small & 1.267 & 0.19 & 1.301 & 0.15 & 1.446 & 0.07 \\
\hline \multicolumn{7}{|l|}{$\begin{array}{l}\text { Exposure to NGO } \\
\text { activities }\end{array}$} \\
\hline Not NGO member(RC) & - & - & - & - & - & - \\
\hline NGO member & 1.325 & 0.09 & 1.393 & 0.05 & 1.364 & 0.11 \\
\hline Access to media & & & & & & \\
\hline Non-exposure(RC) & - & - & - & - & - & - \\
\hline Exposure & 0.794 & 0.24 & 0.832 & 0.29 & 0.814 & 0.29 \\
\hline
\end{tabular}

RC : Reference category, HR : Hazard ratio.

Neonatal mortality: Although maternal education plays significant role in reducing infant and under-five mortality, the study shows that mother's education has no significant effect on neonatal mortality. Kamal et al. (2012) found maternal education as an important risk factor of neonatal mortality in his study using BDHS 2007 data and this findings is contradictory to our analysis. Like under-five mortality and infant mortality, the analysis reveals that the rate of survival during neonatal period is highest in Barisal whereas neonatal of Sylhet experience lowest rate of survival compared to that of Dhaka. 
It has been observed that male children are more likely to die within 28 days of life compared to the female children. This may be due to the reason that naturally male children are more vulnerable than female children from the starting time of pregnancy (BDHS 2014). It is clear from the analysis that neonatal whose size at the time of birth was small experienced $45 \%$ more rate of mortality than those neonatal whose size at birth was large or average. Child's size at birth is significantly associated with neonatal mortality whether it has insignificant impact on both infant and under-five mortality. Although mother's exposure to NGO activities is significantly related with both infant and under-five mortality, it has been found as insignificant determinant of neonatal mortality. Access to media plays no important role in reducing neonatal deaths in Bangladesh.

From the study we observe that maternal education, region, mother's exposure to NGO activities are the potential determinants of infant and under-five mortality whereas region, gender of child, child's size at birth have significant effect on neonatal mortality in Bangladesh. The findings of the study suggest to improve female educational attainment as mother's education lowers the risk of dying of under-five children and infants. Policy makers should focus on the factors responsible for the highest mortality rate in Sylhet. Special health intervention programs should be taken in order to reduce mortality rates of children in Sylhet. Mothers' membership of NGO has been found to be significant determinant for under-five and infant mortality but shows no significant influence on neonatal mortality. NGOs should ensure maternity leave with salary and other facilities that could reduce the rates of mortality of children. Government should take necessary steps to enhance public awareness about maternal health care during pregnancy and child health care which may improve child survival in Bangladesh

\section{Acknowledgement}

The Authors would like to thank National Institute of Population Research and Training (NIPORT) for allowing them to use the BDHS, 2014 dataset in their study.

\section{References}

Bangladesh Demographic and Health Survey (BDHS), 2014. NIPORT, Dhaka, Bangladesh; Mitra and Associates, Dhaka, Bangladesh.

Chowdhury, A. H. 2013. Determinants of under-five mortality in Bangladesh. Open Journal of Statistics 3: 213-219.

Fatima, T.Z. K.A. Mohammad and W. Bari. 2016. Log-Logistic Proportional Odds Model for Analyzing Infant Mortality in Bangladesh. Asia Pacific Journal of Public Health 29(1): 60-69.

Hossain, M. and M. Islam 2008. Socio-economic variables affecting infants and children mortality in Bangladesh. The Internet Journal of Health 9(2). 
Islam, R., M. Hossain, M. Rahman and M. Hossain. 2013. Impact of socio-demographic Factors on child mortality in Bangladesh: An Multivariate Approach. International Journal of Psychology and Behavioral Sciences 3(1): 34-39.

Kamal, S.M. M., M. Ashrafuzzaman and S. A. Nasreen. 2012. Risk factors of neonatal mortality. Journal of Nepal Paediatric Society.32(1): 37-46.

Khadka, K. B. L. S. Lieberman, V. Giedraitis , L. Bhatta and G. Pande. 2015. The socio-economic determinants of infant mortality in Nepal: analysis of Nepal Demographic Health Survey, 2011. BMC Pediatrics .15:152

Ministry of Health and Family Welfare (MOHFW) [Bangladesh]. 2016. Health, Nutrition and Population Strategic Investment Plan 2016-21. Planning Wing, MOHFW, Government of the People's Republic of Bangladesh.

Mondal, N. I., K. Hossain and K. Ali, 2009. Factors influencing infant and child mortality: A case study of Rajshahi District, Bangladesh. Journal of Human Ecology 26(1): 31-39.

Nasejje, J. B., H.G. Mwambi, and T .N. O. Achia. 2015. Understanding the determinants of underfive child mortality in Uganda including the estimation of unobserved householdand community effects using both frequentist and Bayesian survival analysis approaches. BMC Public Health 15:1003.

Nisar, Y. B. and M. J. Dibley. 2014. Determinants of neonatal mortality in Pakistan: secondary analysis of Pakistan Demographic and Health Survey 2006-2007. BMC Public Health $14: 663$.

Rahman, M. 2008. Factors Affecting on Child Survival in Bangladesh: Cox Proportional Hazards Model Analysis.The Internet Journal of Tropical Medicine 6(1).

Siddiqi, N. A., N. Haque and A. Goni. 2011. Differentials and determinants of under-five mortality in Bangladesh. International Journal of Current Research 3(1): 142-148.

United Nations children's fund (UNICEF). 2015. Levels and trends in child mortality. Available at: http://www.unicef.org/media/files/IGME_Report_Final2pdf.

(Revised copy received on 29.11.2017) 